\title{
Polio Across the Iron Curtain: Hungary's Cold War with an Epidemic
}

By Dóra Vargha. Cambridge: Cambridge University Press, 2018. Pp.254.

Dóra Vargha's history of Hungary’s fight against polio in the 1950s combines social, cultural, and political history to demonstrate how Cold War politics were central to this story. She also challenges generally held perceptions of the iron curtain as impenetrable and demonstrates, at least in the case of polio, that the border between east and west was much more porous than generally thought. By highlighting contradictions, such as the global acceptance of Hungary's epidemic control methods at the same time as the Hungarian government was persona non grata in the West, Vargha demonstrates the complicated and paradoxical nature of Hungary's relationship with western countries at this time.

Like many countries, Hungary suffered from a shortage of iron lungs to treat polio patients. Western countries were prepared to donate iron lungs to Hungary, and the Red Cross played a role in sharing iron lungs between countries in what it termed "mutual assistance." Vargha argues that it is too simplistic to see the arrival of iron lungs in Hungary as a technological transfer from west to east. It was a network of assistance, conceived in the West, where all countries were potential givers and takers. When shortages occurred, innovation became necessary, and Hungarian doctors transformed iron lungs into devices that could serve three infants at a time. In another example of innovation, Hungarians produced a machine called the Electrospirator, which became the most widely used device in treating polio in Hungary.

At the center of this history is Hungary's use of the Salk vaccine, and then the Sabin vaccine. Vargha highlights how the Salk vaccine was important beyond the United States as it quickly became used on a global scale, but Hungary struggled to produce it. Then, with a second major outbreak in 1959, the assumed success of the Salk vaccine had to be re-evaluated. 
There was disagreement over the method (intracutaneous or intramuscular) and the amount of vaccine to use. The intracutaneous method used a fraction of the dose. This method was used until Spring 1959, when administration changed to intramuscular. Vargha shows that these decisions were significant, as many Hungarians later blamed the state for the 1959 outbreak, thinking it had halved the required dosage in an act of frugality. While this view remains unsubstantiated, it was a widely held belief and has persisted to this day.

In December 1959, Hungary became one of the first countries in the world to begin mass vaccinations with the Sabin vaccine - four years before the United States, where it had been developed. The development of a live vaccine demonstrated that polio vaccine development was not just an American story, with trials of the Sabin vaccine being conducted across Eastern Europe, including in Hungary. Vargha shows scientists and public health authorities worked together as a global community to stop the polio epidemic; they "transcended Cold War barriers and defied the world order" (p. 161). Science had the potential to unite humanity and stop the Cold War. At the same time, the Sabin vaccine was accepted much more readily in Hungary than the Salk vaccine as trials had been conducted on the "right" side of the iron curtain.

In her final chapter, "After the end of Polio," Vargha asks when epidemics finish. She makes the point that many histories of epidemics have not considered; what happens once the disease is considered to have been eradicated in a particular area? "The end" is not a fixed point, but can depend on one's position. In the Hungarian case, the state "forgot" about polio and ignored polio survivors with disabilities from the 1960s onwards, rendering them invisible. Vargha shows that polio care was seldom discussed or advanced after the 1960s; medical students were no longer taught about the disease and patients were hidden away. Vargha convincingly makes the point that to end the history of polio in Hungary in the early 1960s is both to repeat the mistake of the Hungarian state and medical establishment in ignoring these 
patients and results in misunderstanding the long and enduring nature of polio and other diseases. Vargha extends this argument by providing a nuanced discussion of attempts at eradication of diseases, and the limitations of such attempts. By problematizing the periodization of epidemics, Vargha suggests a new approach for historians of disease and recentres the stories of survivors of epidemics.

This excellent book will interest those working on the history of vaccinations, medicine, and public health more generally as well as those interested in cold war politics and post-war Eastern European history.

\section{Hayley Brown}

Dr. Hayley Brown is a research fellow in the Centre for History in Public Health at the London School of Hygiene and Tropical Medicine. She is currently researching the history of the New Zealand health system and its transnational links. 